\title{
Eco-friendly design and realization of a bioflax bag (BFB) by infusion technology
}

\begin{abstract}
A composite $\mathrm{BfB}$ bag, based on a commercial green epoxy resin and flax textile, has been designed and realized by using vacuum infusion process. Different polymer matrices, natural textiles and respective composites have been preliminary analyzed, manufactured and experimentally characterized in order to set properly the optimal bag materials. Various geometries and accessories have been selected and examined to ensure easy bag opening and to optimize its design allowing demonstrating the feasibility of a novel eco-friendly hand bag by infusion technology that is a low cost process manufacturing usually adopted for naval and automotive components.
\end{abstract}

Keywords: bioepoxy, flax, composites, infusion
Volume 3 Issue I - 2017

\author{
MR Ricciardi,V Antonucci \\ CNR- Institute for Polymers, Composites and Biomaterials, \\ Portici, Italy
}
Correspondence: V Antonucci, CNR- Institute for Polymers, Composites and Biomaterials, National Research Council, $P$. Enrico Fermi, I, 80055 Portici, Italy, Tel 081775 8836, Email vincenza.antonucci@cnr.it

Received: September 20, 2017 | Published: October 10, 2017

\section{Introduction}

Epoxy resins and respective composites are widely used for several industrial applications, ranging from aeronautical, automotive to civil architecture and sportive goods providing many advantages over traditional reinforcing materials such as light weight coupled with high mechanical properties, resistance to aggressive chemical agents and impermeability to water. ${ }^{1-3}$ Other important characteristics of composites include: unlimited capability to be molded and perfect adaptability to the original shape of the structural element that need to be reinforced; non invasive installation, low tooling and machinery costs, possibility to repair the structure without interrupting its utilization by users.

More recently, the development and the adoption of reinforced biocomposites based on natural fibers (flax, jute, hemp, and cotton) have been gained significant research interest due the growing ecological consciousness and new specific legislative requirements regarding environmental protection and recycling of materials. ${ }^{4-5}$ These materials, in fact, come from renewable sources, are easily recoverable or degradable for end use.

Natural fibre-reinforced biocomposites have been especially used for applications where not structural characteristics are required. ${ }^{6}$

Natural fibers compared to traditional synthetic fibers, such as carbon or glass, have many advantages: lower costs, easily accessible and abundant in nature, low chemical risk for operators, biodegradable and biocompatible, recyclable with various matrices, density less than or equal to synthetic fibers, source of income for agricultural producers. The main advantage of natural fibers is related to the low energy required for their production. Composites made with natural fibers have lower values of mechanical properties, such as the tensile module, than those made with synthetic fibers. This difference, however, is mitigated if the specific properties, relative to the density, are considered. Their specific properties, such as rigidity, are, in fact, comparable to those of glass fibers. In addition, natural fibers are about 50\% lighter and cheaper than glass fibers.

Therefore, natural fiber composites currently represent one of the emerging areas in polymer science and technology for various enduse applications. $^{7}$
In this framework, due to their aesthetical features and the high flexibility for tailoring ultimate properties, epoxy biocomposites are attracting also the attention of artists to create fashion accessories or design objects that in some cases are manufactured by using recycled textiles. ${ }^{8}$ In fact, Pil et al. ${ }^{9}$ report recent applications of flax and hemp composites in sporting goods applications, such as tennis rackets, bicycles, fishing rods or ski's or interior design and furniture.

This study is aimed to demonstrate the feasibility of a composite $\mathrm{BfB}$ bag, based on a commercial green epoxy resin and flax textile. The Bfb has been designed by trial and error method and realized by vacuum infusion technology that is a low cost process manufacturing usually adopted for naval and automotive components.

\section{Materials and methods}

Two different commercial low-viscosity polymer systems have been considered for the design of the bag: the polyurethane PX-1000 (PU) by Axson technologies and the Super Sap CLR bioepoxy resin by Entropy resin, which is formulated with biobased renewable materials such as wood pulp and waste coming from bio-fuels production. As reinforcement, cotton and two kinds of flax, $\mathrm{A}$ and $\mathrm{B}$, with different textures have been analyzed.

Composite materials have been realized by using the vacuum infusion technology. ${ }^{10}$ This process basically involves three steps: lay up of a fiber reinforcement, vacuum application and fiber impregnation by a thermoset resin, cure of the resin. The textile is placed onto a one-sided rigid mold, a formable vacuum bag material replaces the common RTM matched metal tool. The resin is injected through one or more inlet gates, depending on part size and shape. Vacuum is applied through a single or multiple vents in order to remove the air from the fiber preform and to drive the fiber impregnation of the part by resin. A resin distribution net medium is placed onto the reinforcement to promote the resin flow, to allow complete wet-out of the preform and to eliminate voids and dry spots.

Both the matrices and respective realized composites have been mechanically tested by using a Dynamic Mechanical Analyzer (DMA-Q 800, TA Instruments, and New Castle, DE) to obtain the stress-strain curve. The tests were carried out under tensile load at room temperature by using a pre-load of 10-2 $\mathrm{N}$ and a ramp force of 
$1.0000 \mathrm{~N} / \mathrm{min}$ up to $18.0000 \mathrm{~N}$. The elastic modulus $\mathrm{E}$ was determined by the initial slope of the stress-strain curve.

Further, the two flax textiles were analyzed by optical microscopy by the polarized light optical Olimpus BX51 Instruments.

\section{Results and discussion}

\section{Mechanical tests}

The experimental DMA tests have been carried out both on the neat investigated polymer systems and on relative reinforced composites samples of size $30 \times 6.5 \times 2 \mathrm{~mm}$. Figure 1 shows the obtained stressstrain diagram for the PU and bioepoxy resin. PU is more rigid than bioepoxy that, due the vegetable oils presence, has an elastic modulus of $592 \mathrm{MPa}$, while PU elastic modulus is $986 \mathrm{MPa}$.

(Figure $2 \& 3$ ) show the DMA results for the cotton based and flax composites respectively. Table 1 reports the calculated elastic modulus data of all tested materials. In general, cotton composites are more rigid than corresponding flax those. Further, due to higher stiffness of PU matrix, the elastic modulus of PU/Cotton composite is $5.5 \mathrm{GPa}$, while Bio epoxy/cotton has a modulus of $4 \mathrm{GPa}$. Thus, the first design of the bag has been conceived by using the bioepoxy resin and the cotton textile, in order to ensure such flexibility to the bag.

Table I Elastic modulus of all tested materials

\begin{tabular}{ll}
\hline Materials & E (MPa) \\
\hline PU & 986 \\
BioEpoxy & 592 \\
PU-Cot & 5570 \\
PU-Flax(A) & 5246 \\
PU-Flax(B) & 4685 \\
BioEpoxy-Cot & 3954 \\
BioEpoxy-Flax(A) & 3889 \\
BioEpoxy-Flax(B) & 3475 \\
\hline
\end{tabular}

\section{Microscopy analysis}

Both flax textiles have been observed by optical microscopy in order to have information about their texture and, hence, to understand the different mechanical behavior. Figures $4 \& 5$ shows the optical observation and the texture schematic of the two textiles, i.e. flax A and flax B respectively.

One should observe that the texture of two textiles is quite different. In particular, flax A seems to be characterized by a plane texture, that has the largest possible number of intersections between warp and weft yarns that alternate one over and the other under, exchanging the position at each turn (Figure 4b). On the other hand, flax B has a diagonal texture, that is obtained by twisting the warp and weft yarns, so that the first warp passes under a weft yarn and then over two and so on. In the subsequent row, there is the same weave, but shifted, so the resulting textile has diagonal rows texture (Figure $5 \mathrm{~b}$ ).

The different texture of the adopted flax textiles can affect the final behavior of the composite materials.. The flax B, having the fibers placed along the $45^{\circ}$ direction, is more flexible and has a lower modulus than flax $\mathrm{A}$, that results more rigid due to the $\left[0,90^{\circ}\right]$ configuration of the fibers, according with the DMA results of Table 1.

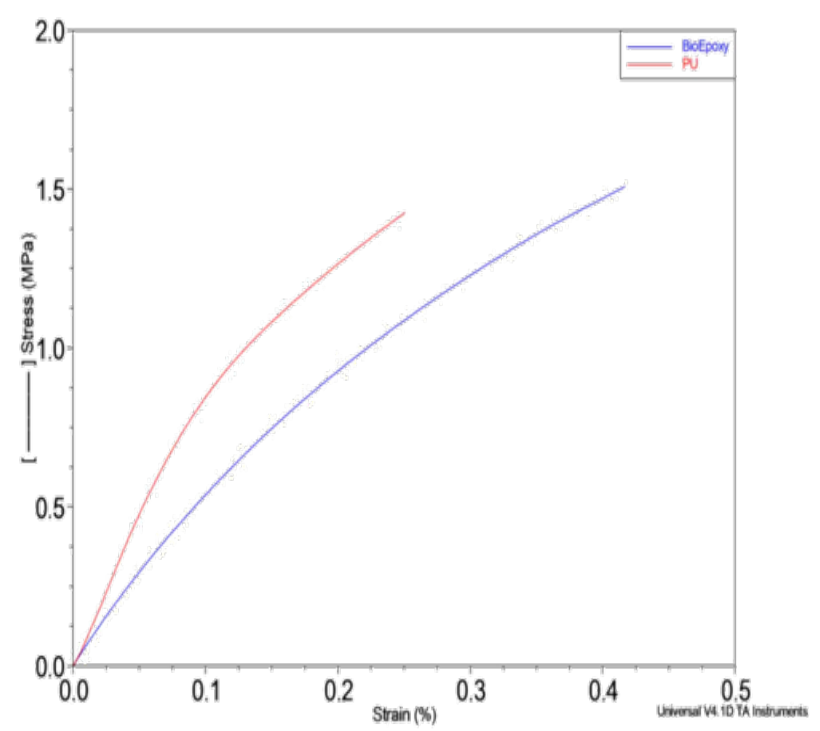

Figure I Stress-strain diagram for PU and bioepeoxy resin.

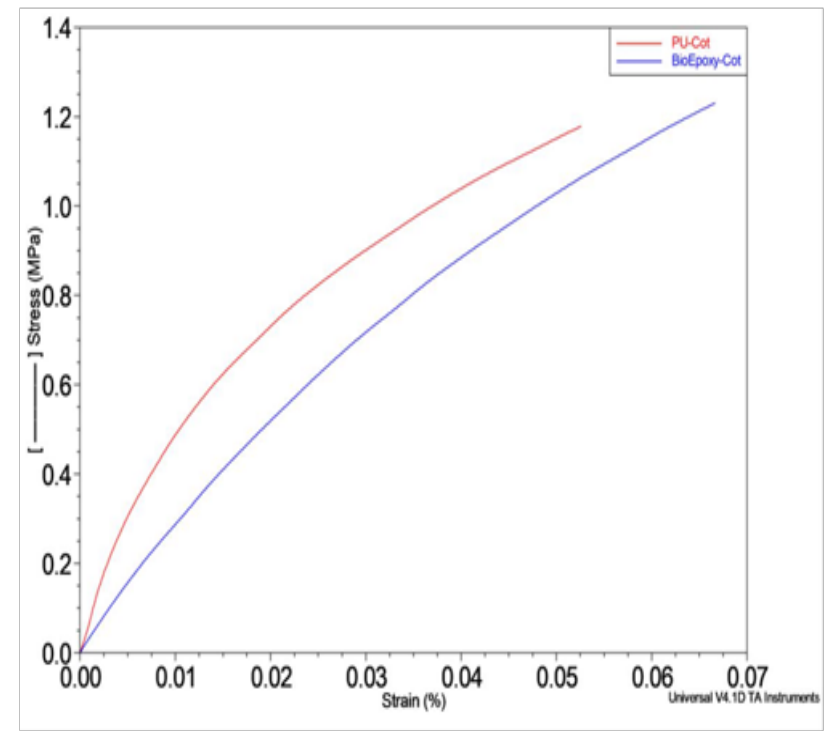

Figure 2 Mechanical results for cotton based composites.

\section{BfB design}

The design of the bag has been performed by trial and error procedure, in order to select the optimal geometry and accessories. Figure 6 shows a sketch of the bag that is characterized by an inner cotton fabric bag, a circular hole as handle and two metal rings to fix both bags.

This geometry has been modified to ease the bag opening. In particular, a semielliptical hole has been considered and instead of the rings, automatic buttons have been used.

\section{$B f B$ realization}

The textiles have been impregnated by using the vacuum infusion process that basically involves three steps: lay up of a fiber reinforcement, vacuum application and fiber impregnation by a thermoset resin, cure of the resin. The textile is placed onto a one-sided rigid mold, a formable vacuum bag material replaces the common 
RTM matched metal tool. Figure 7 shows the tested cotton textile. It's possible to observe that the handle hole has been preliminary cut in order to reduce subsequent finishing operations.

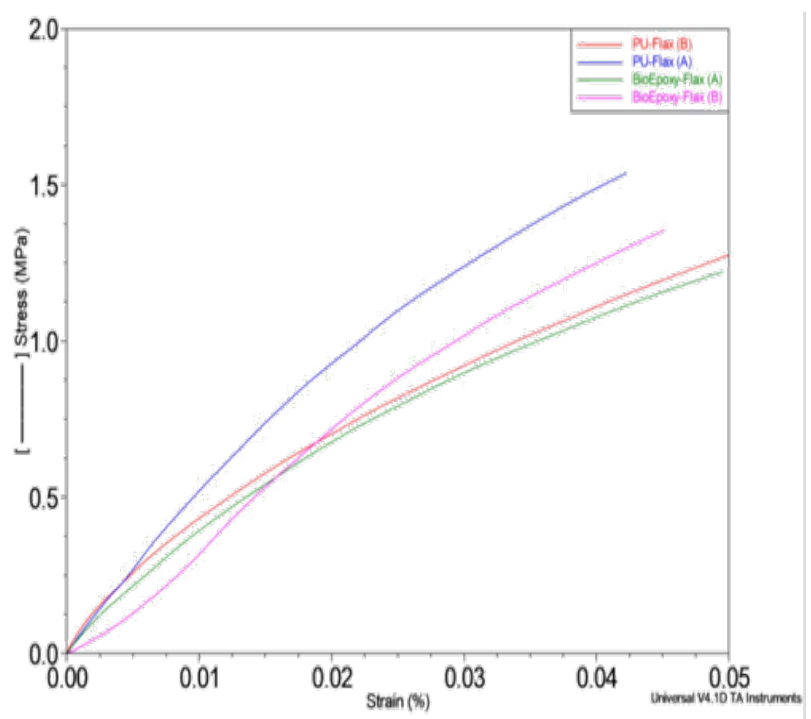

Figure 3 Mechanical results for flax based composites.

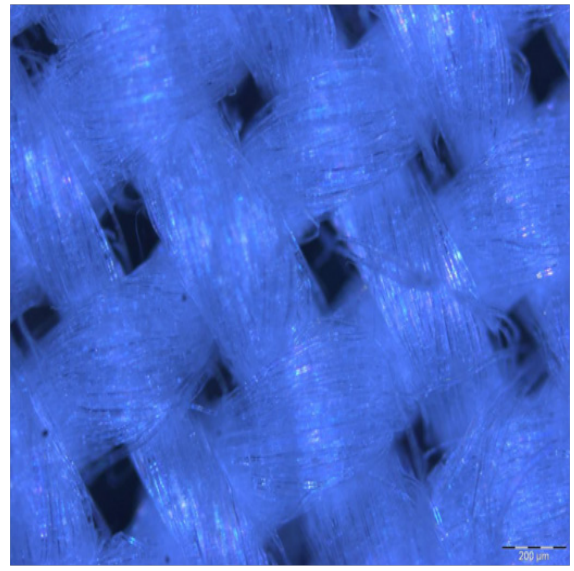

(a)

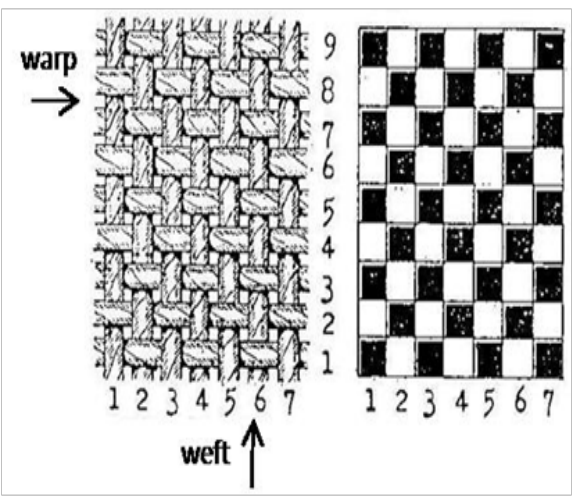

(b)

Figure 4 Flax A microstructure a) optical micrography, b) texture schematic.

The resin is injected through one or more inlet gates, depending on part size and shape. Vacuum is applied through a single or multiple vents in order to remove the air from the fiber preform and to drive the fiber impregnation of the part by resin. A resin distribution net medium is placed onto the reinforcement to promote the resin flow, to allow complete wet-out of the preform and to eliminate voids and dry spots. Figure 8 shows the whole manufacturing assembly.

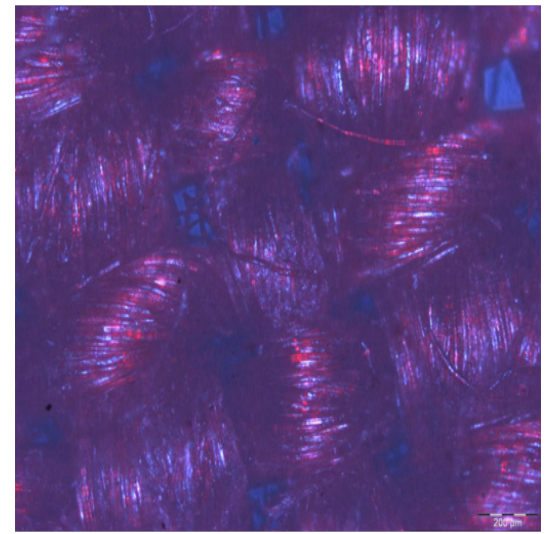

(a)

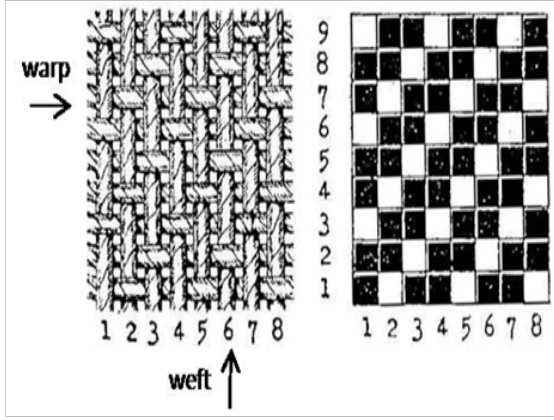

(b)

Figure 5 Flax B microstructure a) optical micrography, b) texture schematic.

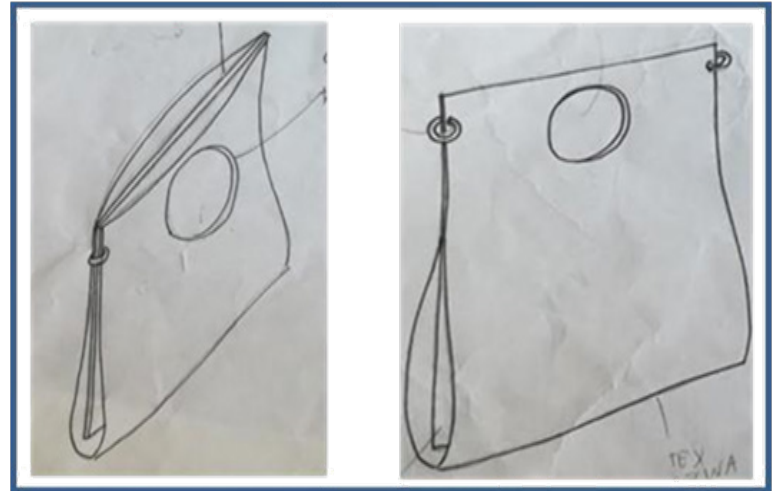

Figure 6 Bag sketch.

Figure 9 shows the realized bag with the first design, based on the circular hole, the bioepoxy resin and the cotton textile. In the preliminary design the inner cotton bag was stitched to the external composite bag. However, this configuration gave some opening difficulties. Therefore, the design was changed by substituting the circular hole with a semielliptical and adopting automatic buttons instead of the rings. In addition, the bag of Figure 9 resulted too rigid, so it was decided to change also the composite material. In particular, the flax B in combination with the bioepoxy resin was chosen, being less rigid than the other material systems, as reported above. 
Figures 10 report the frontal and lateral view of the optimal realized BfB bag that showed a good flexibility and handling.

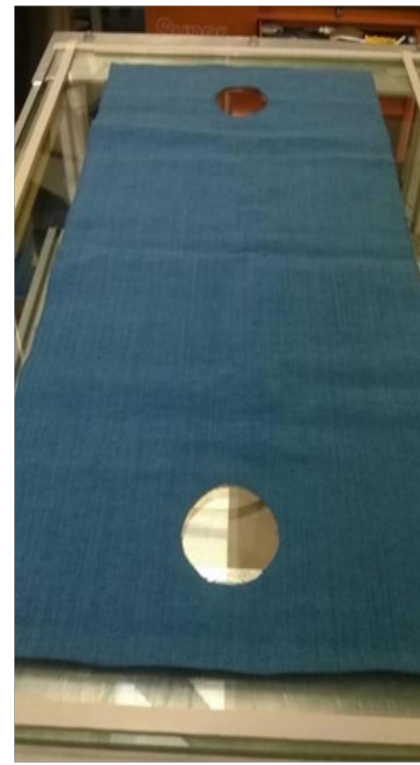

Figure 7 Stacking of the textile.

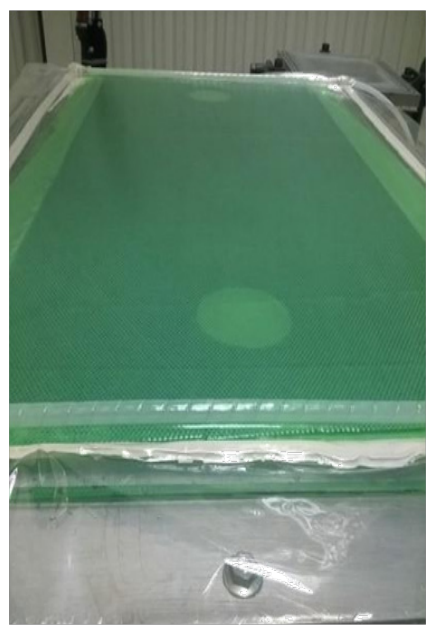

Figure 8 Bagged manufacturing assembly.

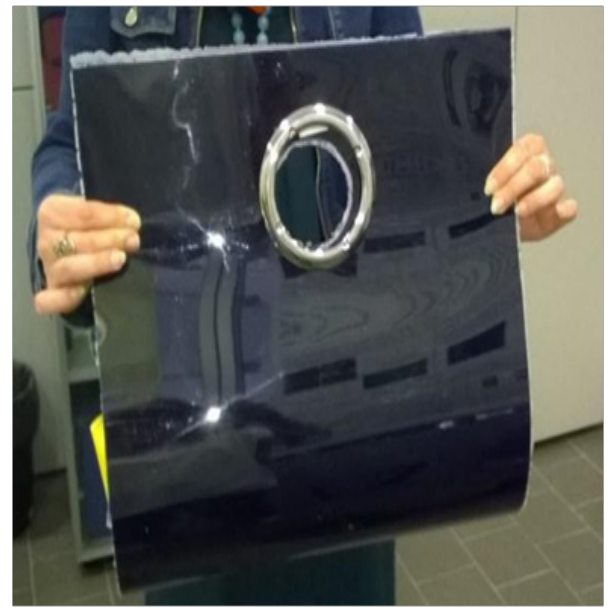

Figure 9 First realized bag with circular hole, based on bioepoxy resin-cotton composite.

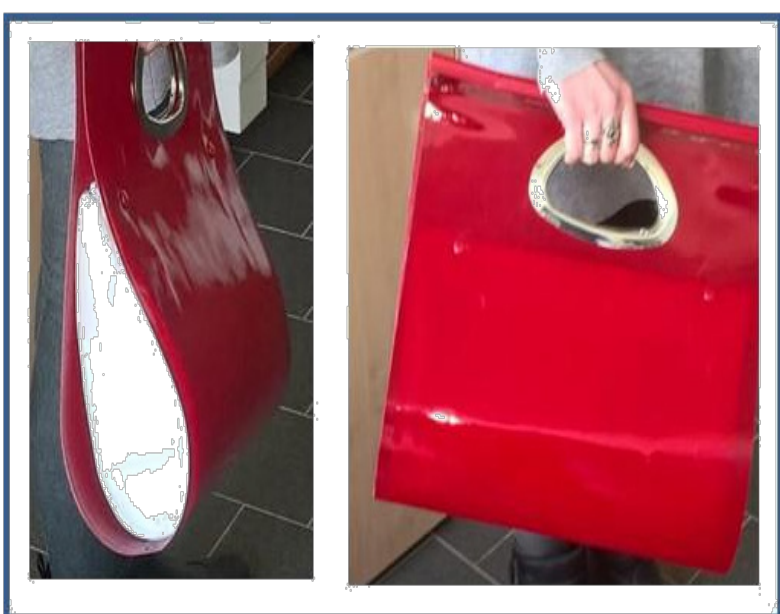

Figure 10 Realized BfB bag with the semielliptical handle (frontal and lateral view), based on bioepoxy resin and flax B composite.

\section{Conclusion}

A composite bag based on natural fiber textiles and a commercial polymer resin has been optimized and realized by investigating different composite constituents, geometries and accessories and accounting for handling and flexibility criteria. The composite materials have been selected after analysis and experimental testing of two different commercial matrix systems: a polyurethane and bioepoxy resin and three types of reinforcement: cotton and two kinds of flax. Vacuum infusion process has been adopted to impregnate the natural textiles demonstrating the feasibility of a fashion accessory by using materials and technologies usually employed in other industrial applications.

\section{Acknowledgements}

Author would thank Dr. Sara Del Vecchio for her contribution to the bag design.

\section{Conflict of interest}

The authors certify that they have NO affiliations with or involvement in any organization or entity with any financial interest or non-financial interest in the subject matter or materials discussed in this manuscript.

\section{References}

1. Ricciardi MR, Martone A, Borriello A, et al. Mechanical behavior of hybrid fiber-reinforced composites manufactured by pulse infusion. Polymer Composites. 2015.

2. Pernice MF, Annicchiarico D, Petriccione A, et al. Structural polymer composite materials for muscle-skeletal diagnostic systems. $J$ Reinforced Plastics \& Composites. 2011;30(4)287-300.

3. Georgios Koronis, Arlindo Silva, Mihail Fontul. Green composites: A review of adequate materials for automotive applications. Composites Part B: Engineering. 2013;44(1):120-127.

4. Mohammed L, Ansari MNM, Pua G, et al. A Review on Natural Fiber Reinforced Polymer Composite and Its Applications. International Journal of Polymer Science. 2015. p. 15. 
5. Gurunathan T, Smita Mohanty, Sanjay K Nayak. A review of the recent developments in biocomposites based on natural fibres and their application perspectives. Composites: Part A Applied Science \& Manufacturing. 2015;77:1-25.

6. Zini E, Scandola M. Green composites: an overview. Polym Compos. 2011;32(12):1905-15.

7. Zonatti WF, Gama Guimarães BM, Duleba W, et al. Thermoset composites reinforced with recycled cotton textile residues. Textiles \& Clothing Sustainability. 2015. p. 1-12.
8. Asim M, Khalina Abdan, Jawaid M, et al. A Review on Pineapple Leaves Fibre and Its Composites. International J Polymer Science. 2015. p. 16.

9. Lut Pil, Farida Bensadoun, Julie Pariset, et al. Why are designers fascinated by flax and hemp fibre composites? Composites Part A: Applied Science \& Manufacturing. 2016;83:193-205.

10. Ricciardi MR, Antonucci V, Durante M, et al. A new cost-saving vacuum infusion process for fiber-reinforced composites: Pulsed infusion. J Composite Materials. 2014;48(11):1365-1373. 